\title{
Metformin-associated lactic acidosis refractory to hemodialysis in the setting of concomitant alcohol intoxication
}

\begin{abstract}
Metformin is widely used as a first-line therapy for type 2 diabetes mellitus (T2DM). Although metformin is generally safe, metformin-associated lactic acidosis (MALA) can occur. Early recognition and treatment of MALA is crucial to prevent complications and mortality.

We present a case of 51-year-old man with hypertension and T2DM who was brought to our emergency room with altered mental status after ingesting $18,000 \mathrm{mg}$ metformin and binge drinking of alcohol the previous night. Laboratory blood analysis showed $22.8 \mathrm{mmol} / \mathrm{L}$ lactic acid, $36.7 \mathrm{mmol} / \mathrm{L}$ anion gap, $6 \mathrm{mmol} / \mathrm{L}$ bicarbonate, $18 \mathrm{mg} / \mathrm{dL}$ blood urea nitrogen, and $2.5 \mathrm{mg} / \mathrm{dL}$ creatinine. Based on blood analysis results, the patient was diagnosed with MALA and acute kidney injury. He underwent several rounds of hemodialysis but ultimately did not survive.

Immediate hemodialysis is indicated for MALA in patients with significant comorbidities. However, hemodialysis may be ineffective to counteract metformin overdose in conjunction with alcohol intoxication. In such cases, depletion of NAD+ stores can result in an inability to clear lactic acid. Severe lactic acidosis associated with metformin treatment has an increased mortality rate in the presence of comorbidities that decrease lactate and metformin clearance.
\end{abstract}

Keywords: metformin overdose, lactic acidosis, alcohol intoxication, acute kidney injury
Volume 5 Issue 6 - 2018

\author{
Jamila A Benmoussa, ${ }^{1,2}$ Benjamin Chaucer, ${ }^{2}$ \\ Marie Chevenon, ${ }^{2}$ Farhang Ebrahimi ${ }^{2}$ \\ 'Department of Endocrinology, St Peter's Hospital,Albany NY, \\ USA \\ ${ }^{2}$ Richmond University Medical Center, USA
}

\section{Correspondence: Jamila A Benmoussa, Department of} Endocrinology, St Peter's Hospital,Albany NY, USA Email jbenmaus@gmail.com

Received: April 22, 2018 | Published: November 15, 2018

\section{Introduction}

Metformin is currently the most frequently prescribed anti-diabetic drug worldwide. Metformin-associated lactic acidosis (MALA) is a rare but well documented side effect of metformin and can result in serious morbidity, and in rare cases, death. Severe lactic acidosis in the emergency department can have many different clinical presentations that compound the difficulty of MALA diagnosis and increase the mortality rate.

\section{Case presentation}

A 51-year-old male with type 2 diabetes mellitus (T2DM) and hypertension was brought by ambulance to our emergency department with altered mental status. The patient was reported to be confused and acting bizarrely. Household contacts disclosed that the night prior to admission the patient had a session of binge alcohol consumption in conjunction with excessive metformin, pioglitazone, and glimepiride intake eight hours prior to presentation. Further investigation revealed that he ingested eighteen $1,000 \mathrm{mg}$ metformin pills (total $18,000 \mathrm{mg}$ ). During triage, his blood glucose level was $16 \mathrm{mg} / \mathrm{dL}$. The patient was given two ampules of dextrose $50 \%$ IV and started on an octreotide drip for refractory hypoglycemia and sulfonylurea overdose. Blood analysis indicated severe metabolic acidosis with $22.8 \mathrm{mmol} / \mathrm{L}$ lactic acid and an anion gap of $36.7 \mathrm{mmol} / \mathrm{L}$. A bicarbonate drip was started. His heart rate then dropped to between 30 and 40 beats per minute and he became hypotensive with a blood pressure of $98 / 42 \mathrm{mmHg}$. The patient went into cardiac arrest, but with cardiopulmonary resuscitation (CPR) he returned to sinus rhythm. The patient was then intubated and transferred to the intensive care unit (ICU). Laboratory findings included a chemistry panel showing $139 \mathrm{mmol} / \mathrm{L}$ sodium, $3.7 \mathrm{mmol} / \mathrm{L}$ potassium, $101 \mathrm{mmol} / \mathrm{L}$ chloride, $6 \mathrm{mmol} / \mathrm{L}$ bicarbonate,
$18 \mathrm{mg} / \mathrm{dL}$ blood urea nitrogen, and $2.5 \mathrm{mg} / \mathrm{dL}$ creatinine. His white blood cell count was elevated to 15.2 x 109 cells/L. Liver function tests showed elevated levels of alanine aminotransferase (90units/L) and $82 \mathrm{units} / \mathrm{L}$ aspartate aminotransferase. Ammonia levels were elevated to $69 \mu \mathrm{mol} / \mathrm{L}$. Prothrombin time was $21.1 \mathrm{sec}$, the partial thromboplastin time exceeded $200 \mathrm{sec}$ and the international normalized ratio was 2.92. Arterial blood gas was $\mathrm{pH} 6.8$ and had $46 \mathrm{mmHg}$ pCO2 and $8.0 \mathrm{mg} / \mathrm{dL}$ bicarbonate. The serum alcohol level was $132 \mathrm{mg} / \mathrm{dL}$; acetaminophen and salicylate levels were negative, as was the urine drug screen. In the ICU, the patient was started on vasopressors to maintain a mean arterial pressure (MAP) $>65$. He underwent four rounds of emergent hemodialysis. Despite immediate dialysis, the blood gas $\mathrm{pH}$ remained below 7.15 , with a $\mathrm{pCO} 2$ of $57 \mathrm{mmHg}$ and $19.9 \mathrm{mg} / \mathrm{dL}$ bicarbonate. Despite high positive end expiratory pressure (PEEP) and high fraction of inspired oxygen (FiO2), his respiratory status deteriorated. After multiple cardiac arrests and resuscitations, the patient passed away.

\section{Discussion}

Metformin is an oral hypoglycemic belonging to the biguanide class that is widely prescribed to treat T2DM. Metformin can reduce diabetes-related morbidities by up to $32 \%$, diabetes-related deaths by up to $42 \%$, and all-cause mortality by up to $36 \%$.

Metformin inhibits gluconeogenesis by reducing expression of genes encoding gluconeogenic enzymes. Metformin also increases both insulin sensitivity of peripheral tissues and glucose uptake while decreasing fatty acid oxidation., ${ }^{2,3}$ Unlike sulfonylureas, thiazolidinediones, and insulin, metformin does not cause weight gain. ${ }^{3}$ Common side effects of metformin include gastrointestinal irritation that manifests as diarrhea, flatulence, nausea and vomiting. 
The incidence of lactic acidosis in patients using metformin is estimated to be 3-10 per 100,000persons/year. ${ }^{4}$ Lactic acidosis associated with biguanides is a clinical concern, as evidenced by the withdrawal of phenformin from the market in 1977 due to the high risk of lactic acidosis associated with this drug. Compared with metformin, phenformin is lipophilic and has a higher affinity for mitochondrial membranes and a more powerful inhibitory effect on the mitochondrial respiratory chain. Phenformin increases lactate release from muscle, inhibit lactate oxidation, and have hepatic metabolism and slow renal excretion. Although metformin is also a biguanide, it does not affect anaerobic lactate production, and the incidence of lactic acidosis with metformin treatment is up to 20 -fold lower than that for phenformin..$^{1,4}$ Metformin is cleared by the kidneys, and its potential for accumulation in patients having decreased renal function could precipitate lactate accumulation. On the other hand, several studies found no association between metformin use and lactic acidosis, although there are reports of increased metabolic acidosis primarily in patients who had at least one condition associated with decreased tissue perfusion (e.g., renal failure, liver disease, heart failure, myocardial infarct, infection, shock, seizure, or alcohol abuse). ${ }^{1,3}$

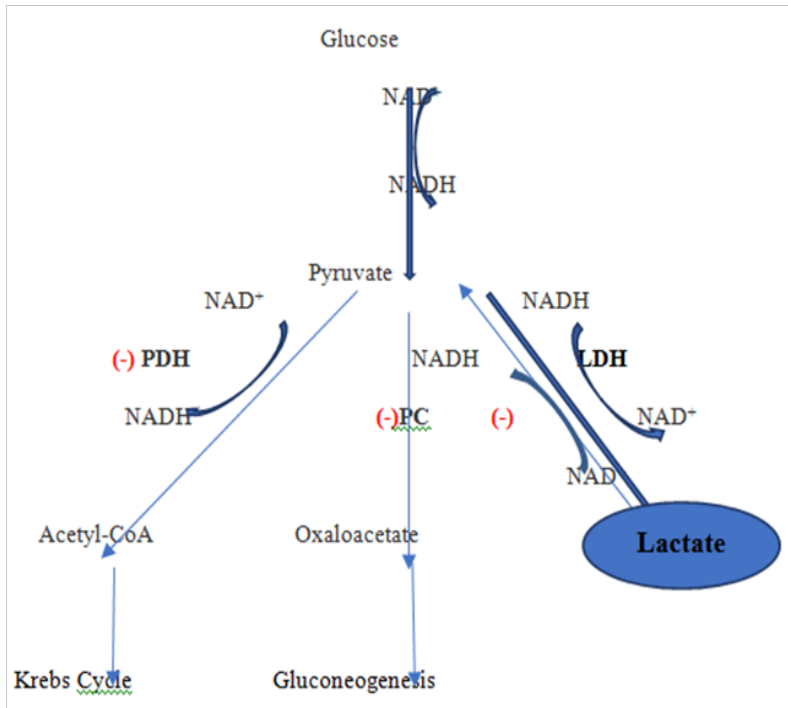

Figure I Metformin induced lactic acidosis and pyruvate metabolism. Metformin has multiple mechanisms of action. Under normal conditions, gluconeogenesis consumes pyruvate through pyruvate carboxylase. Biguanides inhibit pyruvate carboxylase, thereby causing pyruvate to accumulate. Increased amounts of pyruvate are then converted to lactate via lactate dehydrogenase. Conversely, biguanides also inhibit conversion of lactate back to pyruvate for use in gluconeogenesis. ${ }^{13}$

The most studied contraindication of metformin is renal failure. The United States Food and Drug Administration (FDA) advises that metformin may be safely used in patients with mild to moderate renal impairment. This recommendation changed from using serum creatinine concentrations as a single laboratory parameter to use of an equation to calculate an estimated glomerular filtration rate (eGFR), which is a better estimate of renal function. The eGFR considers not only the blood creatinine concentration, but also additional parameters, such as the patient's age, gender, race and/or weight. According to FDA recommendations regarding initiation and discontinuation of metformin based on kidney function, metformin use is contraindicated in patients having an eGFR below $30 \mathrm{~mL} /$ minute $/ 1.73 \mathrm{~m} 2$. Initiation of metformin for patients with an eGFR between $30-45 \mathrm{~mL} /$ minute $/ 1.73 \mathrm{~m} 2$ is also not recommended. Physicians should assess the benefits and risks of continuing treatment for patients taking metformin whose eGFR later falls below $45 \mathrm{~mL} / \mathrm{minute} / 1.73 \mathrm{~m} 2$. Metformin use should be discontinued if the eGFR later falls below $30 \mathrm{~mL} / \mathrm{minute} / 1.73 \mathrm{~m} 2$. Metformin should also be discontinued either at the time of or just before an iodinated contrast imaging procedure in patients who have: 1) an eGFR between 30 and $60 \mathrm{~mL} /$ minute $/ 1.73 \mathrm{~m} 2$; 2) a history of liver disease, alcoholism, or heart failure; or 3) had administration of intra-arterial iodinated contrast ${ }^{5}$. The patient in this case had no history of chronic renal failure, but did have acute kidney injury on presentation.

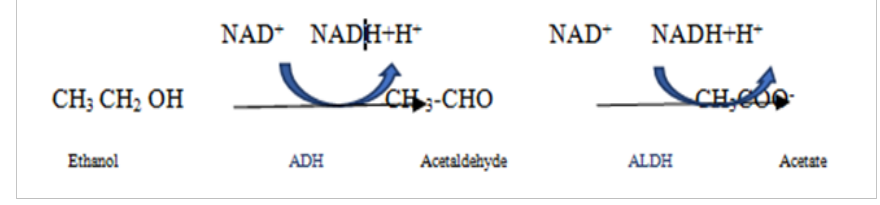

Figure 2 Alcohol metabolism: The chemical name for alcohol is ethanol $\left(\mathrm{CH}_{3} \mathrm{CH}_{2} \mathrm{OH}\right)$. Ethanol in the body is broken down in the liver by alcohol dehydrogenase $(\mathrm{ADH})$, which transforms ethanol into a toxic compound called acetaldehyde $\left(\mathrm{CH}_{3} \mathrm{CHO}\right)$, acetaldehyde it is quickly broken down to a less toxic compound called acetate $\left(\mathrm{CH}_{3} \mathrm{COO}-\right)$ by aldehyde dehydrogenase $(\mathrm{ALDH}) .^{14}$

The amount of metformin required to induce toxicity in acute metformin overdose has not yet been clearly defined. The diagnosis of MALA can be challenging upon presentation given its nonspecific signs and symptoms, and inavailability of metformin levels. ${ }^{6}$ MALA is traditionally defined as lactic acidosis (elevated anion gap metabolic acidosis with $\mathrm{pH}<7.35$ and lactate $>5 \mathrm{mmol} / \mathrm{L}$ ) in a metformin-treated patient. ${ }^{7}$ However, the relationship between metformin accumulation and lactic acidosis is complex, and not all patients with metformin overdose develop lactic acidosis or hyperlactemia. In 2017, Lalau et al. suggested a new classification of lactic acidosis in metformintreated patients, with the following diagnostic categories: 1) 'metformin-unrelated lactic acidosis (MULA)' in patients with low metformin concentration and the presence of other lactic acidosis triggers; 2) 'metformin-induced lactic acidosis (MILA)' exclusively due to metformin; 3) 'metformin-associated lactic acidosis (MALA)' in which both metformin accumulation and systemic diseases contribute to hyperlactatemia; and 4) 'lactic acidosis in metformin therapy (LAMT)' when metformin concentration is not available. ${ }^{8}$ These classifications are not yet widely used.

Between 1960 and 2000, the MALA mortality rate was around $50 \%$ and has since fallen to $25 \% .{ }^{9}$ Although plasma metformin levels do not correlate well with symptom severity, they do correlate with the level of lactic acidosis. ${ }^{10}$ Unfortunately, lactate is an equally unreliable predictor of survival, and there is no connection between lactate concentration and mortality. A ten-year retrospective study performed by Renda et al. showed that among all patients who experienced metformin complications in Italy between 2001 and 2011, 7.2\% died. In this study, mortality was predicted only by normal renal function and low arterial $\mathrm{pH}^{11}$ In the three largest retrospective studies of MALA, neither lactate level nor serum metformin concentration were of prognostic significance in relation to mortality. Interestingly, some survivors had the highest plasma concentrations of metformin. ${ }^{7,11,12}$ In fact; mortality outcomes have been strongly linked with sepsis and multidrug intoxication and the number of triggering factors (e.g., kidney failure, liver failure, alcohol abuse). The presence of one factor was more frequently associated with survival compared to the presence of multiple factors, which was more frequently associated with mortalit. ${ }^{6}$ Analysis of our patient revealed that acute kidney 
injury, alcohol abuse, and multidrug overdose, including metformin, predisposed him to a poor outcome.

Our patient had an acute history of binge drinking the night prior to admission that made him more susceptible to lactic acidosis. Mechanisms by which metformin can induce lactic acidosis have not been clearly elucidated. Under normal conditions, gluconeogenesis consumes pyruvate through pyruvate carboxylase. Metformin inhibits pyruvate carboxylase activity, resulting in increased pyruvate levels. This excess pyruvate is converted into lactate via lactate dehydrogenase. Metformin further inhibits the reconversion of lactate back into pyruvate thus inhibiting gluconeogenesis. ${ }^{13}$ this mechanism becomes overwhelmed when toxic levels of biguanides are ingested and result in lactic acidosis Figure 1. Both mitochondrial and cytosolic metabolism of alcohol results in the conversion of nicotinamide adenine dinucleotide (NAD+) to NADH plus a hydrogen ion $(\mathrm{H}+)$. Alcohol dehydrogenase in the cytosol converts ethanol to acetaldehyde using the two electrons from NAD + in a reduction reaction to produce $\mathrm{NADH}$ and $\mathrm{H}+$ Figure 2. ${ }^{14}$ Mitochondrial metabolism of alcohol involves aldehyde dehydrogenase (ALDH) that converts acetaldehyde into acetate by reducing NAD + to NADH and $\mathrm{H}+$. Saturation of ALDH allows acetaldehyde to escape and cause toxic damage associated with alcohol ingestion. ${ }^{14}$ The simultaneous use of NAD+ by metformin and alcohol can explain the toxic effects of this relationship. The high water solubility of metformin explains the success of hemodialysis that promotes drug clearance in cases of acute toxicity. An acute alcohol binge concurrent with a toxic overdose of metformin would sufficiently deplete the body NAD+ stores, resulting in an inability to clear lactic acid to safe levels, which may explain why subsequent rounds of emergent hemodialysis could not resolve the acid-base imbalance in this patient. Hemodialysis is indicated for MALA in patients with significant comorbidities, the critically ill, arterial blood gas $\mathrm{pH}<7.1$, failure of supportive care, renal insufficiency, and a fluid overloaded state. ${ }^{15}$

Metformin is the most frequently prescribed anti-diabetic drug worldwide and its market continues to grow. The side effects of this drug are becoming better defined as more reports of its side effects emerge, although a lethal metformin dose has not yet been defined in the current literature. The increase in the number of metformin side effects indicates that careful monitoring of patients taking metformin is warranted, particularly for patients who have comorbidities that could affect lactate levels or metformin clearance.

\section{Acknowledgements}

None.

\section{Conflict of interest}

Author declares there is no conflict of interest.

\section{References}

1. Lee EY, Hwang S, Lee YH, et al. Association between Metformin Use and Risk of Lactic Acidosis or Elevated Lactate Concentration in Type 2 Diabetes. Yonsei Med J. 2017;58(2):312-318.

2. Stumvoll M, Nurjhan N, Perriello G, et al. Metabolic effects of metformin in non-insulin-dependent diabetes mellitus. $N$ Engl J Med. 1995;333(9):550-554.

3. DeFronzo RA, Goodman AM. Efficacy of metformin in patients with non-insulin-dependent diabetes mellitus. The Multicenter Metformin Study Group. N Engl J Med. 1995;333(9):541-549.

4. Inzucchi SE, Lipska KJ, Mayo H, et al. Metformin in patients with type 2 diabetes and kidney disease: a systematic review. JAMA. 2014;312(24):2668-2675.

5. FDA Drug Safety Communication: FDA revises warnings regarding use of the diabetes medicine metformin in certain patients with reduced kidney function. 2017.

6. Kajbaf F, Lalau JD. The prognostic value of blood $\mathrm{pH}$ and lactate and metformin concentrations in severe metformin-associated lactic acidosis. BMC Pharmacol Toxicol. 2013;14:22.

7. Lalau JD, Race JM. Lactic acidosis in metformin- treated patients. Drug Saf. 1999; 20(4):377-384.

8. Lalau JD, Kajbaf F, Protti A, et al. Metformin-associated lactic acidosis (MALA): moving towards a new paradigm. Diabetes, Obes and Meta. 2017;19:1502-1512.

9. Kajbaf F, Lalau JD. Mortality rate in so-called metformin-associated lactic acidosis: a review of the data since the 1960s. Pharmacoepidemiol Drug Saf. 2014;23(11):1123-1127.

10. Perrone J, Phillips C, Gaieski D. Occult metformin toxicity in three patients with profound lactic acidosis. J Emerg Med. 2011;40(3):271275 .

11. Renda F. Metformin-Associated Lactic Acidosis Requiring Hospitalization. A National 10 Year Survey and A Systematic Literature Review. Eur Rev Med Pharmacol Sci. 2013;17:45-49.

12. Spiller A, Quadrani. Toxic effects from metformin exposures. Annals Pharmacother. 2004;38(5):776-780.

13. Kopec, Kathryn T, Michael J. et al. Metformin-Associated Lactic Acidosis (MALA): Case Files of The Einstein Medical Center Medical Toxicology Fellowship. J Med Toxicol. 2012;9(1):61-66.

14. Manzo-Avalos, Salvador, Alfredo Saavedra-Molina. Cellular and Mitochondrial Effects of Alcohol Consumption. Int J Environ Res Public Health. 2010;7(12):4281-4304.

15. Nguyen H. Metformin intoxication requiring dialysis. Hemodial Int. 2011;15:68-71. 